\title{
Schillenkamp, Elke
}

\section{Die gemeinsame Zeit zwischen Kindern und ihren Eltern}

Zukunft mit Kindern, Zukunft für Kinder. Der UNICEF-Bericht zur Lage der Kinder in Deutschland im europäischen Kontext. Opladen; Berlin; Toronto : Verlag Barbara Budrich 2017, S. 33-49. - (unicef für jedes Kind)

Quellenangabe/ Reference:

Schillenkamp, Elke: Die gemeinsame Zeit zwischen Kindern und ihren Eltern - In: Zukunft mit Kindern, Zukunft für Kinder. Der UNICEF-Bericht zur Lage der Kinder in Deutschland im europäischen Kontext. Opladen; Berlin; Toronto : Verlag Barbara Budrich 2017, S. 33-49 - URN:

urn:nbn:de:0111-pedocs-207828 - DOI: 10.25656/01:20782

https://nbn-resolving.org/urn:nbn:de:0111-pedocs-207828

https://doi.org/10.25656/01:20782

in Kooperation mit / in cooperation with:

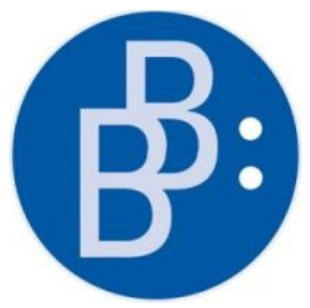

https://www.budrich.de

\section{Nutzungsbedingungen}

Dieses Dokument steht unter folgender Creative Commons-Lizenz:

$\mathrm{http}: / /$ creativecommons.org/licenses/by-nc-nd/3.0/de/deed - Sie dürfen das Werk bzw. den Inhalt unter folgenden Bedingungen vervielfältigen, verbreiten und öffentlich zugänglich machen: Sie müssen den Namen des Autors/Rechteinhabers in der von ihm festgelegten Weise nennen. Dieses Werk bzw. dieser Inhalt darf nicht für kommerzielle Zwecke verwendet werden und es darf nicht bearbeitet, abgewandelt oder in anderer Weise verändert werden.

Mit der Verwendung dieses Dokuments erkennen Sie die Nutzungsbedingungen an.

\section{Terms of use}

This document is published under following Creative Commons-License: http://creativecommons.org/licenses/by-nc-nd/3.0/de/deed.en - You may copy, distribute and transmit, adapt or exhibit the work in the public as long as you attribute the work in the manner specified by the author or licensor. You are not allowed to make commercial use of the work or its contents. You are not allowed to alter, transform, or change this work in any other way.

By using this particular document, you accept the above-stated conditions of use.

\section{Kontakt / Contact:}

peDOcs

DIPF | Leibniz-Institut für Bildungsforschung und Bildungsinformation

Informationszentrum (IZ) Bildung

E-Mail: pedocs@dipf.de

Internet: www.pedocs.de

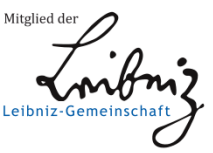


Zukunft mit Kindern, Zukunft für Kinder 

Online-Beitrag von Elke Schillenkamp:

Die gemeinsame Zeit zwischen Kindern und ihren Eltern

aus:

Hans Bertram (Hrsg.)

\section{Zukunft mit Kindern, \\ Zukunft für Kinder}

Der UNICEF-Bericht zur Lage der Kinder in Deutschland im europäischen Kontext

Verlag Barbara Budrich

Opladen • Berlin • Toronto 2017 
Bibliografische Information der Deutschen Nationalbibliothek

Die Deutsche Nationalbibliothek verzeichnet diese Publikation in der Deutschen Nationalbibliografie; detaillierte bibliografische Daten sind im Internet über http://dnb.d-nb.de abrufbar.

(C) 2017 Dieses Werk ist im Verlag Barbara Budrich erschienen und steht unter folgender Creative Commons Lizenz: http://creativecommons.org/licenses/by-nc$\mathrm{nd} / 3.0 / \mathrm{de} /$

Verbreitung, Speicherung und Vervielfältigung erlaubt, kommerzielle Nutzung und Veränderung nur mit Genehmigung des Verlags Barbara Budrich

\section{\begin{tabular}{|c|} 
(C) \\
SOME RIIAFIS RESERRED
\end{tabular}}

Dieses Buch steht im Open-Access-Bereich der Verlagsseite zum kostenlosen Download bereit (https://doi.org/10.3224/84740551).

Eine kostenpflichtige Druckversion (Printing on Demand) kann über den Verlag bezogen werden. Die Seitenzahlen in der Druck- und Onlineversion sind identisch.

\section{ISBN 978-3-8474-0551-1 (Paperback) \\ eISBN 978-3-8474-0499-6 (eBook)}

Gedruckt auf säurefreiem und alterungsbeständigem Papier.

Alle Rechte vorbehalten.

(C) 2017 Verlag Barbara Budrich, Opladen, Berlin \& Toronto www.budrich-verlag.de 


\section{Die gemeinsame Zeit zwischen Kindern und ihren Eltern}

Wie Eltern die gemeinsame Zeit mit ihren Kindern verbringen und welche Bewertung diese gemeinsame Zeit aus der Perspektive der Kinder und Eltern erfährt, wird in diesem Beitrag anhand der neuesten Ergebnisse des GEOlino-UNICEF-Kinderwertemonitors untersucht. Wie wirkt sich die Berufstätigkeit auf das gemeinsame familiale Freizeitverhalten aus, wie wird dieses dann geschlechts- und altersspezifisch beurteilt und welchen Einfluss haben gemeinsame Aktivitäten auf die Zufriedenheit mit dem Familienleben? All diese Fragen werden aufgegriffen und führen schließlich zu einem hochaktuellen Diskurs darüber, was unter ,guten Eltern“ eigentlich zu verstehen ist.

\section{Einleitung}

In regelmäßigen Abständen gibt das Kinderhilfswerk der Vereinten Nationen zusammen mit der Kinderzeitschrift GEOlino das Forschungsprojekt „GEOlino-UNICEF-Kinderwertemonitor" in Auftrag, um den Kindern in Deutschland zu verschiedenen relevanten Themen eine Stimme zu verleihen. Nach Erhebungen in den Jahren 2006, 2008 und 2010 wurde die Studie 2014 erneut durchgeführt (UNICEF 2010). ${ }^{1}$

Für die repräsentative Befragung wurden Ende 2013 und Anfang 2014 ähnlich wie in den Vorjahren 1.012 Kinder im Alter von sechs bis 14 Jahren und ihre mit ihnen zusammenlebenden Mütter zu Hause durch eine/n Interviewer_in mithilfe eines standardisierten Fragenkatalogs befragt. Erstmalig sind zudem auch die Väter befragt worden, sodass ein ganzheitliches Bild der Familie erfasst werden konnte. Auch 2014 stellte die Ermittlung von relevanten Werten und Grundeinstellungen von Kindern und ihren Eltern einen Schwerpunkt der Befragung dar. Zusätzlich wurde analog zu 2010 ebenfalls ermittelt, wie sich die gemeinsame Zeit zwischen den Kindern und ihren Müttern bzw. Vätern gestaltet. Um diesen Aspekt wird es im folgenden Beitrag gehen. Zunächst liegt dabei im Fokus, wie viel Zeit miteinander verbracht wird und wie diese gemeinsame Zeit gestaltet wird. Des Weiteren kann ein dezidiertes Bild darüber gegeben werden, wie die gemeinsame Zeit zwischen Kind und Mutter sowie Kind und Vater von den einzelnen Gruppen subjektiv bewertet wird. Wird sie als genügend empfunden? Und ist es generell eine „schöne“ Zeit? Gerade im Vergleich der einzelnen Paarungen zeichnen sich teils unterschiedliche und interessante Wahrnehmungen ab. Auch aktuell diskutierte Themen, inwieweit sich beispielsweise eine Berufstätigkeit der Mutter auf die gemeinsame Zeit mit dem Kind auswirkt, werden näher beleuchtet.

1 Dies erfolgte in Zusammenarbeit mit der Humboldt-Universität zu Berlin sowie durch Förderungen des Bundesministeriums für Bildung und Forschung und der Robert-Bosch-Stiftung. Mit der Durchführung und Auswertung des Forschungsprojektes wurde das Marktforschungsinstitut Ipsos beauftragt. 


\section{Die gemeinsame Zeit in Zahlen und in der subjektiven Wahrnehmung}

Werden Mütter und Väter danach befragt, wie viele Stunden sie, unterteilt nach Wochenende und Wochentags, mit ihrem Kind verbringen, zeigt sich erwartungsgemäß ein höchst unterschiedliches Bild. Am Wochenende geben Mütter an, durchschnittlich ca. 8,2 Stunden pro Tag mit ihrem Kind zu verbringen. Die Väter kommen im Schnitt auf 6,3 Stunden pro Tag. Die Unterschiede sind unter der Woche entsprechend größer. Mütter schätzen ihre gemeinsame Zeit mit dem Kind von Montag bis Freitag auf durchschnittlich ca. 5,2 Stunden pro Tag, während die Väter bei durchschnittlich 2,9 Stunden liegen. Unter dieser Zeitspanne ist nicht nur aktiv gestaltete Zeit mit dem Kind zu verstehen, d.h. nicht nur die Zeit, in der bewusst etwas gemeinsam unternommen wird. Ein Teil der Zeit gestaltet sich auch so, dass das Kind und der Elternteil zwar Zeit zusammen verbringen, aber parallel unterschiedliche Tätigkeiten ausführen. So kann es durchaus sein, dass die Mutter oder der Vater die Küche aufräumt, während das Kind am Küchentisch liest oder malt.

Wie hoch der Anteil der aktiv gemeinsam verbrachten Zeit ist, variiert je nach Elternteil und betrachtetem Zeitraum stark. Unter der Woche wird bei 35\% der Mütter mindestens die Hälfte der gemeinsamen Zeit aktiv gestaltet. Bei den Vätern ist dies nur bei $20 \%$ der Fall. Am Wochenende steigt der Anteil der Mütter auf 54\% und der der Väter auf $44 \%$. Der Vater holt auch in diesem Zusammenhang am Wochenende auf und beschäftigt sich im Vergleich zu unter der Woche häufiger und auch vermehrt aktiv mit dem Kind. Insgesamt verbringt aber die Mutter mehr Zeit mit dem Kind und gestaltet diese - zumindest unter der Woche - auch häufiger aktiv.

Dabei muss berücksichtigt werden, dass $90 \%$ der befragten Väter in Vollzeit berufstätig sind. Weitere $2 \%$ arbeiten in Teilzeit. Die ausstehenden $8 \%$ geben an, entweder als Hausmann tätig oder arbeitslos zu sein. Von den Müttern sind $70 \%$ berufstätig, davon ein Drittel in Vollzeit und zwei Drittel in Teilzeit. Ein gutes Fünftel der Mütter gibt an, Hausfrau zu sein. Ob eine Berufstätigkeit in Vollzeit oder Teilzeit vorliegt, haben die Eltern selbst eingeschätzt, es wurden keine konkreten Grenzen für die Arbeitszeit vorgegeben. Um somit die Antworten der Mütter und Väter miteinander vergleichen zu können, ist es sinnvoll, die Mütter abhängig von ihrer Berufstätigkeit näher im Detail zu betrachten. Die Hausfrau verbringt an einem Wochentag durchschnittlich 6,7 Stunden mit ihrem Kind; bei der berufstätigen Mutter sind es 4,6 Stunden. Wird zudem unterschieden, ob die Mutter in Vollzeit oder in Teilzeit berufstätig ist, sind es 4,1 Stunden bzw. 4,9 Stunden. Damit liegt auch die berufstätige Mutter weiterhin mit einigem Abstand vor den 2,9 Stunden, die der Vater durchschnittlich mit dem Kind verbringt. Unabhängig davon, ob berufstätig oder nicht, die Mutter bleibt rein unter dem zeitlichen Aspekt von den Eltern die relevantere Bezugsperson des Kindes.

Zusätzlich interessant ist der Vergleich zwischen den Müttern, die mit dem Vater des Kindes oder einem anderen Partner zusammenleben, und denen, die alleinerziehend sind. Letztere, unabhängig davon, ob berufstätig oder nicht, verbringen an einem Werktag immerhin noch im Durchschnitt 4,8 Stunden mit ihrem Kind. Bei den Müttern, die einen Partner an ihrer Seite haben, ist es mit 5,2 Stunden nur marginal mehr Zeit. An einem Wochenendtag kommen beide Gruppen sogar auf die identische Anzahl von 8,2 Stunden. Somit hat die Tatsache, dass eine Mutter alleinerziehend ist, keinen Einfluss auf die Anzahl der Stunden, die sie gemeinsam mit ihrem Kind verbringt. Es ist anzunehmen, dass eine alleinerziehende Mutter eher weniger Zeit für sich selbst in Anspruch nimmt oder in andere Dinge investiert, bevor sie die gemeinsame Zeit mit ihrem Kind kürzt. 


\section{Kinder / Mütter / Vater Gesamt - Mengeneinschätzung gemeinsame Zeit \\ Angaben in \%, am Wochenende}

Gemeinsame Zeit: Mutter \& Kind
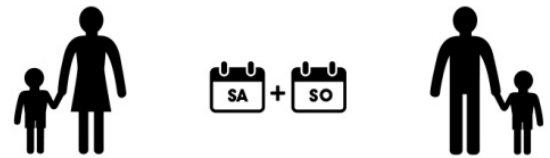

Gemeinsame Zeit: Vater \& Kind

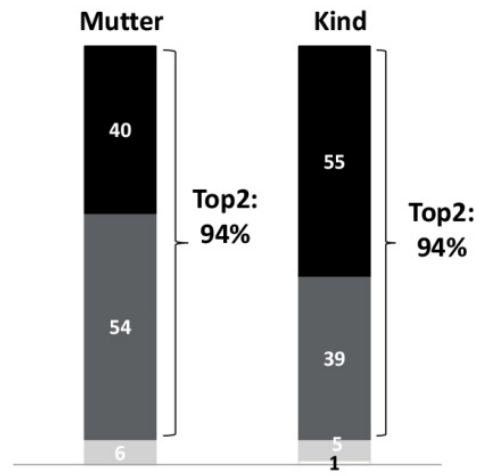

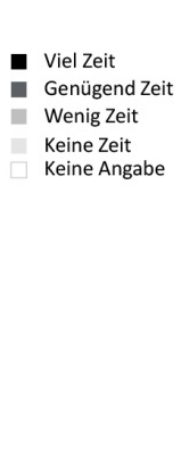

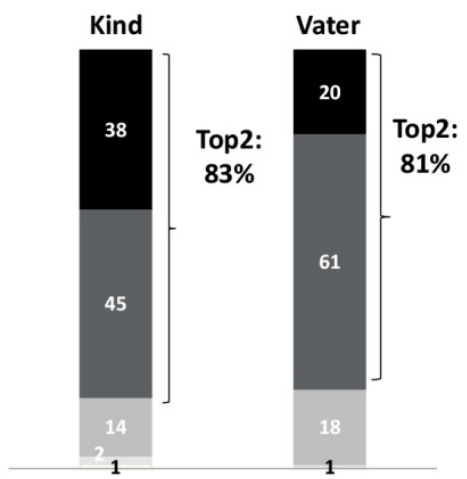

Basis: alle Kinder $n=1.012$, alle Mütter $n=1.002$; alle Väter $n=846$; geschlossene Frage

Abbildung 1: Gemeinsame Zeit von Eltern und Kind am Wochenende

Die Einschätzung der absoluten Menge an Zeit, die mit dem Kind verbracht wird, ist natürlich nur die eine Seite der Medaille. Mindestens genauso wichtig ist die Betrachtungsweise, ob diese Menge subjektiv als ,ausreichend“ empfunden wird, denn an dieser Stelle spielen persönliche Wahrnehmungen eine große Rolle, die aber entsprechend individuell auch den Grad der Zufriedenheit bestimmen. Sowohl die Mütter und Väter als auch die Kinder jeweils einmal bezogen auf ihre Mutter und einmal auf ihren Vater - wurden gefragt, ob sie die gemeinsame Zeit, erneut unterteilt nach Wochentags und Wochenende, als ,viel“, ,,ausreichend“, „wenig“ oder „nicht vorhanden“ beschreiben. Dabei sind in der jeweiligen Konstellation immer nur die Kinder berücksichtigt worden, die auch mit ihrer Mutter bzw. ihrem Vater zusammenleben. Es zeigt sich, dass sich die Elternteile und die Kinder bei ihrer Beschreibung für das Wochenende sehr einig sind (s. Abbildung 1) und diese zudem sehr positiv ausfällt. Zwar beschreiben die Kinder grundsätzlich die Situation noch positiver als ihre Eltern, aber dies ist nicht überraschend, da Erwachsene grundsätzlich etwas kritischer sind. Zudem haben die meisten Eltern wohl unterschwellig eher das Gefühl, zu wenig Zeit für bestimmte Dinge, vor allem aber für ihre Kinder, zu haben.

Für das Wochenende geben jeweils $94 \%$ der Kinder und der Mütter an, dass sie „viel“ bzw. ,genügend“ Zeit miteinander verbringen. Wie bereits erwähnt, sind die Kinder sogar noch positiver in ihrer Einschätzung, denn während 55\% der Kinder von „,viel Zeit“" sprechen, sind es bei den Müttern 40\%. Zwar sinkt das Niveau der positiven Aussagen bei der Konstellation der Väter und ihren Kindern etwas, aber auf beiden Seiten beschreiben mehr als vier Fünftel die gemeinsame Zeit als „,viel“ bzw. „genügend“. Auch hier ist das Elternteil weitaus kritischer als das Kind, da nur $20 \%$ der Väter die gemeinsame Zeit mit „viel“ beschreiben, während es $38 \%$ der Kinder sind. Die Väter sind in diesem Zusammenhang sehr kritisch mit sich, denn sie verbringen immerhin auch einiges weniger an Zeit mit ihren 
Kindern als die Mütter. Dies scheint ihnen durchaus bewusst zu sein. Immerhin geben knapp $18 \%$ der Väter an, am Wochenende „wenig Zeit“" mit ihren Kindern zu verbringen. Aber auch dieses Bild wird ähnlich von den Kindern gespiegelt, denn $14 \%$ der Kinder sprechen von ,wenig Zeit“" mit ihrem Vater und weitere $2 \%$ von „,keiner Zeit“".

Wenn der Fokus auf die Woche gelegt wird, verändert sich das Bild etwas (vgl. Abb. 2). Bei der Konstellation Mutter und Kind sinkt die Top2-Box, d.h. die Zustimmung mit „viel“ und ,genügend“ Zeit, leicht auf $81 \%$ aus Sicht der Mutter bzw. auf $85 \%$ aus der Sicht der Kinder. Somit empfinden immer noch mehr als vier Fünftel der Mütter und Kinder die gemeinsame Zeit von Montag bis Freitag als „,viel“ bzw. „genügend“. Auffällig ist aber, dass am Wochenende mehr als die Hälfte der Kinder (55\%) die gemeinsame Zeit als „viel“" beschrieben haben. Unter der Woche sind es 31\%, jedoch mehr als die Hälfte der Kinder (54\%) wählt die Beschreibung ,genügend“. Die Bewertung ist hier also auch bei den Kindern etwas kritischer.

Gerade unter der Woche zeigen sich zudem Unterschiede in der Bewertung der Kinder, je nachdem ob ihre Mutter berufstätig ist oder als Hausfrau daheim ist. Ist letzteres der Fall, beschreiben $93 \%$ der Kinder die Zeit als „,viel“ bzw. „genügend“. Sobald die Mutter in Teilzeit berufstätig ist, sind es $89 \%$, das Niveau bleibt damit noch vergleichbar. Wenn die Mutter aber in Vollzeit arbeitet, nutzen noch gut zwei Drittel $(69 \%)$ der Kinder die Beschreibung „viel“ bzw. „genügend“. $29 \%$ der Kinder von in Vollzeit berufstätigen Mütter geben an, dass die Mutter ,wenig Zeit“ für sie hat. Die identische Entwicklung, erneut auf einem kritischeren Niveau, zeigt sich auch bei den Angaben der Mütter: Sobald sie in Vollzeit berufstätig sind, geben $39 \%$ der Mütter an, dass sie „wenig Zeit“ für ihr Kind haben. Entsprechend ihrer Mutterrolle würden sie gerne mehr Zeit mit ihren Kindern verbringen, können dies aber aufgrund ihrer Berufstätigkeit nur schwer ermöglichen.

\section{Kinder / Mütter / Vater Gesamt - Mengeneinschätzung gemeinsame Zeit Angaben in \%, werktags}
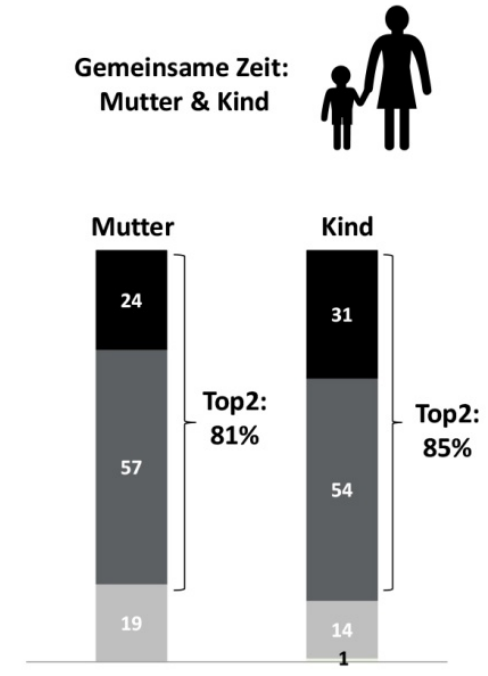
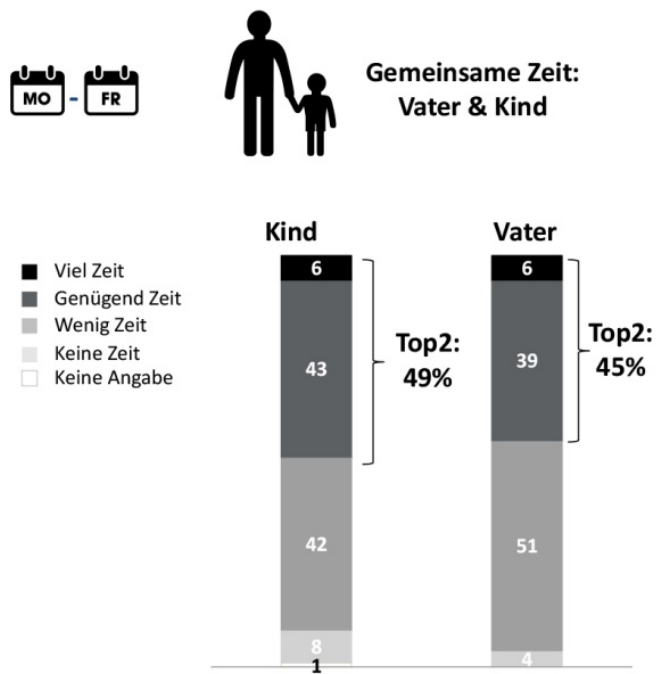

Basis: alle Kinder $n=1.012$; alle Mütter $n=1.002$; alle Väter $n=846$; geschlossene Frage

Abbildung 2: Gemeinsame Zeit von Eltern und Kind unter der Woche 
Da die Mehrzahl der befragten Väter in Vollzeit berufstätig ist, verwundert es nicht, dass die Bewertung der gemeinsamen Zeit zwischen Kindern und Vätern unter der Woche sehr viel kritischer ausfällt. Lediglich knapp die Hälfte der Kinder (49\%) und Väter (45\%) beschreiben die Menge an Zeit als „,viel“ bzw. „genügend“. Die Hälfte der Väter geben an, dass sie unter der Woche ,wenig Zeit“" mit ihren Kindern verbringen. $8 \%$ der Kinder geben sogar an, dass ihr Vater unter der Woche ,keine Zeit“ für sie hat. Grundsätzlich kann festgehalten werden, dass ein sehr übereinstimmendes Bild über die Menge an gemeinsamer Zeit zwischen dem Kind und dem jeweiligen Elternteil besteht: Die Situation wird ähnlich beschrieben, sodass auch vermutet werden kann, dass über diese Thematik in den Haushalten gegebenenfalls offen gesprochen wird. Es sind unter den Familienmitgliedern keine verzerrten Wahrnehmungen festzustellen, die zu Missverständnissen führen könnten.

\section{Freizeitverhalten der Kinder: Tätigkeiten und Spielpartner}

Bei den Freizeitaktivitäten, die Kinder zwischen sechs und 14 Jahren täglich oder mehrmals die Woche ausüben, zeigt sich ein heterogenes Bild zwischen Mediennutzung und anderen Tätigkeiten (vgl. Tabelle 1).

\begin{tabular}{|c|c|c|c|c|c|c|}
\hline & & \multirow[b]{2}{*}{ Total } & \multicolumn{2}{|c|}{ Alter } & \multicolumn{2}{|c|}{ Geschlecht } \\
\hline & & & $6-10$ & $11-14$ & Jungen & Mädchen \\
\hline Basis & & 1012 & 557 & 455 & 511 & 501 \\
\hline 1. & Fernsehsendungen schauen & $95 \%$ & $95 \%$ & $94 \%$ & $95 \%$ & $94 \%$ \\
\hline 2. & Freunde treffen und spielen oder sich unterhalten & $82 \%$ & $83 \%$ & $81 \%$ & $82 \%$ & $82 \%$ \\
\hline 3. & Musik hören & $81 \%$ & $76 \%$ & $87 \%$ & $75 \%$ & $87 \%$ \\
\hline 4. & Draußen spielen & $73 \%$ & $84 \%$ & $60 \%$ & $77 \%$ & $69 \%$ \\
\hline 5. & Faulenzen/nichts tun & $63 \%$ & $57 \%$ & $69 \%$ & $61 \%$ & $64 \%$ \\
\hline 6. & Filme anschauen, Hörbücher/-spiele hören & $58 \%$ & $63 \%$ & $52 \%$ & $58 \%$ & $59 \%$ \\
\hline 7. & Bücher/Zeitschriften lesen & $56 \%$ & $60 \%$ & $51 \%$ & $47 \%$ & $65 \%$ \\
\hline 8. & Mit meinen Spielsachen spielen (Lego, Playmobil...) & $53 \%$ & $72 \%$ & $30 \%$ & $54 \%$ & $52 \%$ \\
\hline 9. & Mich mit Tieren beschäftigen (Haustier) & $51 \%$ & $50 \%$ & $52 \%$ & $46 \%$ & $55 \%$ \\
\hline 10. & Computerspiele spielen/Spielekonsole & $50 \%$ & $45 \%$ & $55 \%$ & $66 \%$ & $33 \%$ \\
\hline 11. & Klettern, Toben & $49 \%$ & $66 \%$ & $29 \%$ & $53 \%$ & $46 \%$ \\
\hline 12. & Sport machen/Sportverein & $44 \%$ & $39 \%$ & $49 \%$ & $48 \%$ & $40 \%$ \\
\hline 13. & Malen/Basteln/Modellbau & $41 \%$ & $57 \%$ & $21 \%$ & $34 \%$ & $48 \%$ \\
\hline 14. & Im Internet sein (surfen, für die Schule recherchieren) & $41 \%$ & $23 \%$ & $63 \%$ & $40 \%$ & $42 \%$ \\
\hline 15. & Schreiben (Briefe/Tagebuch, E-Mail) & $30 \%$ & $23 \%$ & $39 \%$ & $23 \%$ & $38 \%$ \\
\hline 16. & Communities/soziale Netzwerke im Internet besuchen... & $28 \%$ & $11 \%$ & $50 \%$ & $27 \%$ & $30 \%$ \\
\hline 17. & Gesellschaftsspiele/Brettspiele spielen & $25 \%$ & $34 \%$ & $13 \%$ & $26 \%$ & $23 \%$ \\
\hline 18. 1 & Mit der Familie etwas unternehmen, z.B. Ausflüge & $20 \%$ & $23 \%$ & $16 \%$ & $19 \%$ & $21 \%$ \\
\hline 19. $s$ & Selber Musik machen, Instrument spielen/üben & $18 \%$ & $18 \%$ & $18 \%$ & $16 \%$ & $21 \%$ \\
\hline 20. 5 & Sammeln, mit einer Sammlung beschäftigen & $17 \%$ & $20 \%$ & $12 \%$ & $18 \%$ & $16 \%$ \\
\hline 21. $s$ & Shoppen gehen, Bummeln & $10 \%$ & $9 \%$ & $11 \%$ & $6 \%$ & $13 \%$ \\
\hline 22. $s$ & Sportveranstaltungen besuchen & $7 \%$ & $7 \%$ & $6 \%$ & $7 \%$ & $6 \%$ \\
\hline 23. 1 & Musikkonzerte besuchen/ins Museum oder ins Theater gehen & $1 \%$ & $1 \%$ & $1 \%$ & $1 \%$ & $1 \%$ \\
\hline
\end{tabular}

Tabelle 1: Freizeitaktivitäten der 6-14-jährigen Kinder (täglich und mehrmals die Woche)

Es schauen $95 \%$ aller Kinder täglich oder mehrmals die Woche „Fernsehsendungen“. Es folgt „Freunde treffen“, was von $82 \%$ der 6-14-Jährigen in der gleichen Frequenz unternommen wird. Sowohl bei „Fernsehsendungen schauen“ als auch bei „Freunde treffen“ gibt es keine relevanten Unterschiede in den Altersgruppen 6-10 und 11-14 Jahre oder zwischen 
den Geschlechtern. Währenddessen wird „Musik hören“, mit $81 \%$ bei allen Kindern an dritter Stelle, etwas häufiger von den etwas älteren Kindern zwischen 11-14 Jahren und auch eher von Mädchen angegeben. Mit etwas Abstand rangiert an vierter Stelle „draußen spielen“: Nahezu drei von vier Kindern spielen täglich oder mehrmals die Woche draußen. Auch hier zeigen sich Unterschiede im Alter, denn bei den jüngeren Kindern zwischen sechs und zehn Jahren steigt der Wert auf $84 \%$ an. Die Top5-Liste der Nennungen wird ergänzt durch ,faulenzen/nichts tun“ (63\%), etwas stärker durch die älteren Kinder $(69 \%)$ getrieben. Es folgen nach Fernsehen und Musik weitere Medien wie „Filme anschauen/ Hörbücher hören“ (58\%) und „Bücher/Zeitschriften lesen“ (56\%). Letzteres wird häufiger von Mädchen (65\%) unternommen als von Jungen (47\%).

Auf den Plätzen 7-10 liegen „mit Spielsachen spielen“ (53\%), ganz stark getrieben durch die jüngeren Kinder $(72 \%)$, „mit Tieren beschäftigen“ (51\%), wiederum etwas stärker bei den Mädchen (55\%), sowie „Computerspiele spielen“" (50\%); letzteres - nicht überraschend - etwas weiter vorne bei den älteren Kindern (55\%) und ganz klar bei den Jungen $(66 \%)$. Bei denen ist „Computerspiele spielen“ sogar in der Top5-Liste der regelmäßigen Freizeitaktivitäten vertreten. Unter allen Kindern folgen erst auf Platz 14 ,im Internet sein (surfen, für die Schule recherchieren)“ (41\%) und auf Platz 16 „Communities/soziale Netzwerke im Internet besuchen“ (28\%). Diese beiden Aktivitäten werden aber im Vergleich der Altersgruppen interessant und daher an dieser Stelle noch einmal gesondert herausgegriffen: 11-14-Jährige üben beide Tätigkeiten weitaus häufiger aus (,im Internet sein“ mit $63 \%$, „soziale Netzwerke“ mit 50\%). „Mit der Familie etwas unternehmen, z.B. Ausflüge" liegt etwas abgeschlagen auf dem 18. Platz, aber dies ist auch keine Tätigkeit, die mit einer entsprechenden Häufigkeit von täglich oder mehrmals die Woche punkten kann. Immerhin unternimmt jedes dritte Kind einmal pro Woche etwas gemeinsam mit der Familie. Bei weiteren $40 \%$ der Kinder finden Familienausflüge seltener als einmal pro Woche statt.

Aber natürlich werden nicht nur Familienausflüge gemeinsam mit den Eltern unternommen. Auch einzelne der anderen Freizeitaktivitäten werden sehr häufig entweder mit beiden Elternteilen oder einem einzelnen zusammen verbracht. Von den zehn häufigsten Freizeitaktivitäten wird die erste, „Fernsehsendungen schauen“, von knapp jedem zweiten Kind $(43 \%)$ gemeinsam mit beiden Eltern unternommen. Jedes vierte Kind sitzt eher gemeinsam mit der Mutter vor dem Fernseher. Bei 5\% ist es eher der Vater. „Filme anschauen/Hörbücher anhören" wird von knapp einem Drittel der Kinder mit beiden Eltern gemacht. $21 \%$ der Kinder unternehmen dies gemeinsam mit ihrer Mutter, $4 \%$ mit ihrem Vater. Die weiteren Tätigkeiten, die unter den zehn häufigsten Freizeitaktivitäten aufgeführt sind, werden eher selten mit den Eltern gemeinsam unternommen. In diesem Zusammenhang treten Aktivitäten, die eher nicht so häufig unternommen werden, in den Vordergrund. Familienausflüge schneiden hier natürlich am besten ab: $79 \%$ der Kinder unternehmen diese mit ihren beiden Eltern oder zumindest einem Elternteil. Das bedeutet gleichzeitig, dass solche Familienausflüge auch immerhin bei knapp jedem 5. Kind ausschließlich mit anderen Familienmitgliedern wie z.B. den Großeltern stattfinden.

Weitere Freizeitaktivitäten, die von mehr als der Hälfte der Kinder mit den Eltern unternommen werden, sind „Gesellschaftsspiele/Brettspiele spielen“, „Shoppen gehen, bummeln“, ,,Sportveranstaltungen besuchen“ und „Musikkonzerte/Museum/Theater“. Auch hier zeigen sich durchaus verschiedene Rollen, die vom Vater und der Mutter übernommen werden: So ist „Shoppen gehen, bummeln“ eindeutig eine Tätigkeit, die vor allem von der Mutter mit den Kindern unternommen wird. „Gesellschaftsspiele“, „Sportveranstaltungen“ und auch „Musikkonzerte/Museum/Theater“ sind Aktivitäten, die eher als Familie gemeinsam unternommen werden. Es scheint, als wenn besondere Aktivitäten, die nicht so häufig stattfinden, vornehmlich mit beiden Eltern geplant werden. Es ist davon auszugehen, dass 
solche Aktivitäten zu speziellen Anlässen oder als sogenannte „Quality Time“ genutzt werden, in der man gemeinsam etwas Besonders unternimmt. Es fällt auf, dass die meisten Freizeitaktivitäten entweder am häufigsten mit beiden Eltern unternommen werden, oder wenn nur ein Elternteil dabei ist - dies ganz eindeutig die Mutter ist. Dies ist nach der Beschreibung der Menge an gemeinsamer Zeit zwischen Eltern und ihren Kindern im ersten Teil dieses Kapitels nicht überraschend. Einzig bei „Sportveranstaltungen besuchen“ und „Computerspiele spielen“ kann der Vater sich in der Einzelbetrachtung vor die Mutter setzen. Somit zeigt sich an dieser Stelle erneut, dass die hauptsächliche Bezugsperson der Kinder die Mutter ist.

Ein weiterer Trend, der sich zeigt, ist ebenfalls nicht überraschend: Der Anteil der Kinder, die Freizeitaktivitäten gemeinsam mit ihren Eltern unternehmen, sinkt mit ansteigendem Alter. So sind es bei „Fernsehsendungen schauen“ noch 79\% der 6-10-Jährigen, die gemeinsam mit den Eltern oder einem Elternteil vor dem Fernsehgerät sitzen. Bei den 1114-Jährigen sind es nur noch $68 \%$. Ähnliche Tendenzen zeigen sich auch beim „Bücher/ Zeitschriften lesen“. Hier befinden sich die jüngeren Kinder noch im Lernprozess und üben diese Tätigkeiten mit höherem Alter zunehmend alleine aus. Bei vielen Aktivitäten ist der Abnabelungsprozess von den Eltern besonders in den ersten Jahren zu erkennen. Ist der Anteil der gemeinsamen Tätigkeit mit den Eltern bei den 6-7-Jährigen noch sehr hoch, sinkt er kontinuierlich bei den 8-9- sowie den 10-11-Jährigen. Ab 12 Jahren werden bereits viele der Tätigkeiten entweder alleine oder mit anderen Spielpartnern unternommen.

Natürlich ist dies in der Entwicklung der Kinder hin zu eigenständigen Persönlichkeiten nicht erstaunlich und auch notwendig. Allerdings kann im Folgenden gezeigt werden, wie wichtig die gemeinsamen Aktivitäten mit den Eltern für das sensible Beziehungsgeflecht zwischen Kindern und ihren Eltern sind.

\section{Bewertung der gemeinsamen Zeit}

Werden Kinder und die jeweiligen Elternteile danach gefragt, wie sie die gemeinsame Zeit am ehesten beschreiben würden - „meistens schön“, „manchmal schön, manchmal nicht“ oder ,meist nicht so schön“ -, zeigt sich erneut ein sehr übereinstimmendes Bild (vgl. Abb. 3).

Bei der Konstellation Mutter und Kind ist für jeweils $72 \%$ die gemeinsame Zeit „meistens schön“. Die ausstehenden $28 \%$ verteilen sich sowohl bei den Müttern als auch bei den Kindern nahezu ausschließlich auf die Beschreibung „manchmal schön, manchmal nicht“. Lediglich $1 \%$ der Kinder nimmt die gemeinsame Zeit mit der Mutter als „meist nicht so schön“"wahr. Ein ähnliches Bild zeigt sich auch in der Konstellation Vater und Kind: Die Wahrnehmung ist ebenfalls sehr positiv, wenn auch leicht niedriger als bei Mutter und Kind. Gut zwei Drittel $(67 \%)$ der Kinder beschreiben die gemeinsame Zeit mit dem Vater als ,meistens schön“. Bei den Vätern sind es sogar drei Viertel (76\%). Während das ausstehende Viertel der Väter die Beschreibung „,manchmal schön, manchmal nicht“ wählt, sind es bei den Kindern $3 \%$ mit der Beschreibung ,meist nicht so schön“. In Summe zeigt sich damit auch hier ein sehr stimmiges und positives Bild. Die Wahrnehmung innerhalb der Familien stimmt nahezu überein. 
Kinder / Mütter / Vater Gesamt - Bewertung gemeinsame Zeit

Angaben in \%, Gesamtbetrachtung

Gemeinsame Zeit: Mutter \& Kind

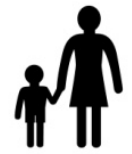

Mutter

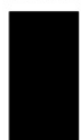

72

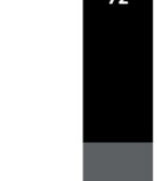

28
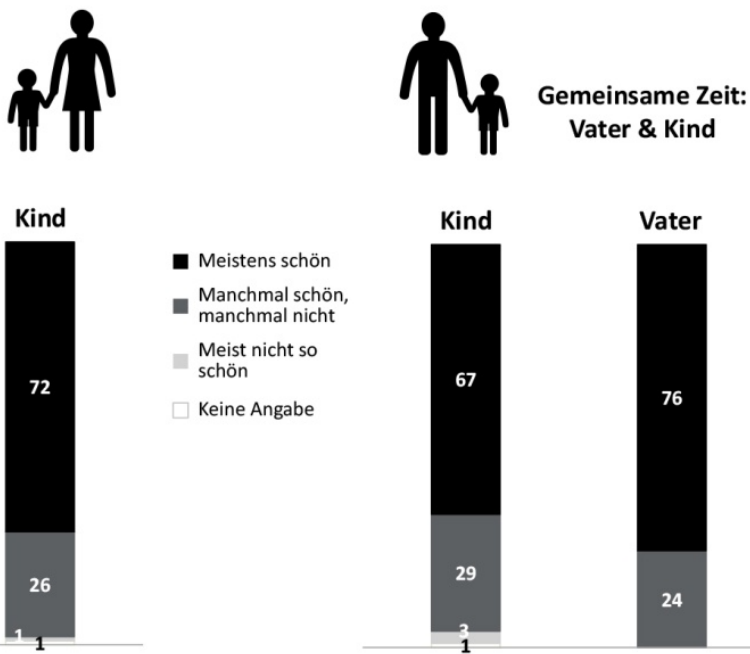

Vater \& Kind

Basis: alle Kinder $n=1.012$; alle Mütter $n=1.002$; alle Väter $n=846$; geschlossene Frage

Abbildung 3: Bewertung der gemeinsamen Zeit durch Eltern und ihre Kinder

Nicht überraschend zeigt sich zudem ein Zusammenhang zwischen der Bewertung der Menge der gemeinsamen Zeit und dem Gefallen der gemeinsamen Zeit. Wenn die Menge an gemeinsamer Zeit aus Sicht des Kindes mit „viel Zeit“ beschrieben wird, ist auch der Anteil derer, die die Zeit als „meistens schön“ beschreiben, höher. Dieser Anteil sinkt, wenn die gemeinsame Zeit mit ,wenig“ beschrieben wird. So beschreiben von den Kindern, die die gemeinsame Zeit mit ihrer Mutter als ,wenig“ empfinden, nur noch $43 \%$ diese als „,meistens schön“. Bei der Wahrnehmung von ,viel Zeit“ sind es immerhin noch $83 \%$. Ähnlich sieht es bei der Konstellation Vater und Kind aus. Unter den Kindern, die die gemeinsame Zeit mit „,viel“" beschreiben, empfinden $83 \%$ diese als „meistens schön“. Wenn die gemeinsame Zeit als „,wenig“ eingeschätzt wird, sinkt dieser Anteil um 21 Prozentpunkte auf $62 \%$. Auch bei den Müttern besteht dieser Zusammenhang, allerdings nicht ganz so stark ausgeprägt. Der Anteil der Mütter mit der Wahrnehmung „meistens schön“ sinkt von $80 \%$ bei „,viel Zeit“ auf $67 \%$ bei „wenig Zeit“. Noch geringer fallen die Unterschiede bei den Vätern aus, denn hier liegen lediglich neun Prozentpunkte zwischen den Gruppen „viel Zeit“ und „wenig Zeit“. Es ist anzunehmen, dass Erwachsene eher zwischen der Menge an gemeinsamer Zeit und der Beschreibung als schön oder nicht schön unterscheiden können. Für Kinder, gerade die etwas jüngeren, hängt die Qualität der gemeinsamen Zeit gegebenenfalls noch stärker mit der Quantität zusammen bzw. kann nicht unterschieden werden.

Aber Kinder können auch weitere konkrete Aspekte benennen, warum sie die gemeinsame Zeit mit ihren Eltern als ,meistens schön“ beschreiben. Die Gründe sind zudem sehr ähnlich und unabhängig davon, ob sich auf die gemeinsame Zeit mit der Mutter oder mit dem Vater bezogen wird. An erster Stelle, von ca. 70\% der Kinder, die die gemeinsame Zeit mit der Mutter bzw. dem Vater als „meistens schön“ beschreiben, genannt, stehen 
gemeinsame Tätigkeiten und Erlebnisse. Das Spektrum dieser gemeinsamen Aktivitäten ist breit gefächert und sehr individuell geprägt. Am häufigsten genannt wird aus Sicht des Kindes sowohl bei der gemeinsamen Zeit mit der Mutter als auch mit dem Vater ,gemeinsames Spielen wie z.B. Brettspiele“ oder ,gemeinsame Ausflüge“. Wenn das Kind an die gemeinsame Zeit mit der Mutter denkt, werden auch Gründe wie ,zusammen shoppen/ einkaufen gehen“, „gemeinsames Fernsehen/Filme schauen“ oder „zusammen kochen, backen oder grillen" häufiger genannt. Im Zusammenhang mit der gemeinsamen Zeit mit dem Vater kann eher „gemeinsam Sport machen“ oder „,gemeinsam toben“ punkten. Es zeigen sich an der einen oder anderen Stelle Unterschiede, die den altbekannten Geschlechterrollen zuzuschreiben sind: Mit der Mutter geht das Kind eher einkaufen bzw. kocht es gemeinsam, während mit dem Vater eher herumgetobt wird.

Ähnlich zu interpretierende Unterschiede fallen ebenfalls auf, wenn die zahlreichen Einzelnennungen bei den Gründen für die schöne gemeinsame Zeit betrachtet werden. So gibt es Nennungen wie z.B. ,auf den Spielplatz gehen“ oder ,gemeinsam im Internet surfen", die vom Kind bei beiden Elternteilen ab und an genannt werden. Andere Einzelnennungen sind konkret einem Elternteil zugeordnet: z.B. „,zusammen Fingernägel lackieren“ eher der Mutter, ,gemeinsames Autowaschen“ eher dem Vater. Gleichzeitig wird aber inzwischen auch ein Erlebnis wie „Mutter bzw. Vater zur Arbeit begleiten zu dürfen“ vom Kind im Zusammenhang mit beiden Elternteilen ab und zu erwähnt. In der Summe wird deutlich, dass Tätigkeiten, die von den Eltern möglicherweise als Kleinigkeiten oder Alltagsroutine wahrgenommen werden, beim Kind durchaus positiv behaftet hängenbleiben und zu einer entsprechenden Grundstimmung führen. Dies bietet für Eltern zahlreiche Ansatzpunkte, ihren Kindern spannende Erlebnisse zu bieten, die in den Köpfen ihrer Kinder lange und positiv konnotiert erinnert werden.

Neben diesen gemeinsamen Aktivitäten/Erlebnissen sind es zwischenmenschliche Gründe, die die mit den Eltern verbrachte Zeit für die Kinder schön werden lassen. Diese Gründe spielen für die Kinder im Zusammenspiel mit der Mutter eine etwas wichtigere Rolle als beim Vater, aber in beiden Konstellationen sind sie der zweitwichtigste Aspekt für ein als angenehm empfundenes Zusammensein. Unabhängig von Mutter oder Vater ist in diesem Zusammenhang „Spaß haben“ am wichtigsten, gefolgt von ,über alles reden können“ und „kuscheln“. Bei der gemeinsamen Zeit mit der Mutter folgt noch ,zuhören/mich ernst nehmen“ und sogar ,zusammen Hausaufgaben machen“ mit einer relevanten Anzahl an Nennungen, während im Zusammenhang mit dem Vater die Antwort ,wenn er Witze macht/albern ist" stärker ausgeprägt ist.

Die Wichtigkeit der übergeordneten Aspekte Gemeinsame Aktivitäten/Erlebnisse und Zwischenmenschliches spielt in den Altersgruppen der 6-10-Jährigen eine andere Rolle als bei den 11-14-Jährigen. In beiden Altersgruppen sind zwar die gemeinsamen Aktivitäten/Erlebnisse der wichtigste Grund, aber bei den etwas Älteren sind die zwischenmenschlichen Aspekte nahezu genauso wichtig. Dies mag zum einen damit zusammenhängen, dass kleinere Kinder auf die Nachfrage, was der Grund für einen Zustand ist, eher konkrete Aktivitäten nennen als übergeordnete Gefühlszustände. Aber gleichzeitig ist im Rahmen des Abnabelungsprozesses der etwas älteren Kinder auch nicht zu unterschätzen, dass gemeinsame Aktivitäten mit den Eltern eher in den Hintergrund rücken, während die Gefühlsebene, inwieweit man sich von den Eltern verstanden und gestärkt fühlt, an Relevanz zunimmt.

Bei den eher negativen Beschreibungen, warum denn die gemeinsame Zeit mit den Eltern auch mal nicht so schön ist, stehen erziehungsrelevante Aspekte im Vordergrund. Von den Kindern werden an dieser Stelle vor allem Gründe wie ,wenn Mutter bzw. Vater auch mal schimpft/meckert“, ,wenn man laut wird“, ,wenn ich im Haushalt mithelfen soll/aufräumen soll“, ,wenn ich Hausaufgaben machen soll“ oder ,wenn mir kaum et- 
was/nichts erlaubt wird“" genannt. Aus Kindersicht sind diese Gründe zwar nachvollziehbar, bieten aber natürlich für die Eltern kaum Optimierungspotenzial, um die gemeinsame Zeit noch schöner werden zu lassen. In diesem Zusammenspiel ist es unumgänglich, dass Kinder von Eltern an der ein oder anderen Stelle bevormundet werden, um erzieherische Prinzipien durchzusetzen. Die Kinder befinden sich in einem Lernprozess, in dem immer auch Dinge angenommen werden müssen, die gegebenenfalls auf wenig Verständnis stoßen.

Im Gegensatz zur Konstellation Mutter und Kind spielen bei der Bewertung der gemeinsamen Zeit mit dem Vater für die Kinder neben den erziehungsrelevanten Aspekten nachgelagert auch zwischenmenschliche Gründe eine Rolle bei der negativen Bewertung. Aspekte, die hier genannt werden, sind z.B. ,wenn er gestresst ist“ oder „wenn er zu wenig Zeit für mich hat". Dies muss allerdings vor dem Hintergrund interpretiert werden, dass der Großteil der Väter in Vollzeit berufstätig ist. Insofern sind Detailbeschreibungen wie z.B., dass der Vater morgens ganz früh aufstehen muss und daher nachmittags zu müde ist, um mit dem Kind zu spielen, oder dass es zwar schön ist, mit ihm zusammen zu sein, aber er dann häufig hektisch ist, weil er so wenig Zeit hat, durchaus nachvollziehbar. Diese Wahrnehmungen wird es aufseiten der Kinder immer geben. Es wird aber an späterer Stelle noch detailliert darauf eingegangen, dass die Kinder durchaus auch Verständnis dafür haben, dass der Vater arbeiten gehen muss.

Neben der Sicht der Kinder haben auch die Elternteile angegeben, was genau an der gemeinsamen Zeit mit dem Kind schön bzw. gegebenenfalls auch nicht so schön ist. Mehr als $80 \%$ der Mütter und Väter nennen positive Aspekte bei der Beschreibung der gemeinsamen Zeit. Wie bei den Kindern spielen die gemeinsamen Tätigkeiten/Erlebnisse hierbei die größte Rolle. Die Eltern sind in der Beschreibung weniger präzise und geben Gründe wie „etwas gemeinsam unternehmen“ oder „etwas gemeinsam spielen“ an. Des Weiteren sind es vor allem übergeordnete Aspekte wie „man genießt die gemeinsame Zeit“, ,gemeinsam Spaß haben“ oder „wir verstehen uns einfach gut“. Zwar eine untergeordnete, aber durchaus relevante Rolle spielt aber auch ein Grund wie „,das Kind ist glücklich/fühlt sich wohl“". Dies spiegelt erneut die Besonderheit der Elternrolle wider: Man selbst nimmt sich zurück und das Wohlergehen des Kindes steht absolut im Vordergrund.

Aber natürlich gibt es auch aus Elternsicht nicht nur Schönes aus der gemeinsamen Zeit mit dem Kind zu berichten. Knapp jeweils 30\% der Mütter und Väter führen negative Aspekte auf. Hierbei gibt es keinen einzelnen Grund, der besonders häufig genannt wird, vielmehr scheint dies in der Wahrnehmung sehr subjektiv zu sein, sodass eine Vielzahl an verschiedenen Gründen angegeben wird. Einzelne Beispiele hierfür sind „die gemeinsame Zeit ist oft stressig/anstrengend“, „Konflikte/Streitereien mit dem Kind“, „schwieriger Umgang mit dem Kind“, „,Kind ist oft unausgeglichen/bockig“, „Kind hat seinen eigenen Kopf". Hier ist anzunehmen, dass solche Reaktionen der Kinder verstärkt auftreten, wenn erziehungsrelevante Maßnahmen durch die Eltern unternommen werden.

In diesem Zusammenhang stellt sich die Frage, was Eltern eigentlich unter ,guten Eltern" verstehen. Von beiden Elternteilen werden dabei ähnliche Bausteine genannt (vgl. Abbildung 4). Am häufigsten werden Erläuterungen wie „für das Kind da sein“ genannt. Da spielen weitere Aspekte wie „hinter ihnen stehen“, ,zu ihnen halten“ und ,,ihnen Rückhalt geben" mit hinein. Kinder sind dabei, ihre Persönlichkeit zu entwickeln, und ihr Selbstbewusstsein muss zunächst aufgebaut werden. Dabei spielt es eine elementare Rolle, dass sie das Gefühl haben, ihre Eltern sind immer für sie da, egal was passiert. Bei den Müttern treten hierbei interessanterweise Unterschiede auf, je nachdem ob sie die Befragung zusammen mit ihrer Tochter oder ihrem Sohn gemacht hat. Dieser Aspekt wird von $31 \%$ der Mütter genannt, wenn parallel auch ihre Tochter befragt wurde. Wenn aber der Sohn befragt wurde, nennen nur 25\% der Mütter diesen Aspekt. Anscheinend spielt dies für Mütter bei Töchtern eine leicht größere Rolle als bei Söhnen. 
Bei der Mutter folgt als zweitwichtigster Aspekt „seine Kinder lieben“. Dies spielt auch bei den Vätern eine relevante Rolle, muss sich aber anderen Aspekten unterordnen. Auch „Kindern Geborgenheit geben“, ebenfalls eher emotional, wird von Müttern häufiger genannt als von den Vätern. Gefühlsaspekte sind bei den Müttern demnach um einiges präsenter als bei den Vätern. Weitere Nennungen wie „ein gutes Vorbild sein“, „sich für Kinder Zeit nehmen“, „Kindern Grenzen aufzeigen“ und „auf die Bedürfnisse der Kinder eingehen“ tauchen bei Müttern und Vätern nahezu gleich häufig auf. Lediglich „Kinder ernst nehmen“ und „Kinder (bei Problemen) unterstützen“ sind wiederum Gründe, die von den Müttern häufiger genannt werden. Insgesamt zeigt sich aber ein sehr übereinstimmendes Bild zwischen den Müttern und Vätern - der Tenor, was unter „guten Eltern“ verstanden wird, ist sehr ähnlich und bildet eine ausgewogene Grundlage bei der Erziehung und dem Miteinander mit dem Kind.

\section{Mütter / Vater Gesamt - Beschreibung „gute Eltern“}

Offene Abfrage, alle Nennungen mit mind. 12\% Nennungen bei einer Zielgruppe

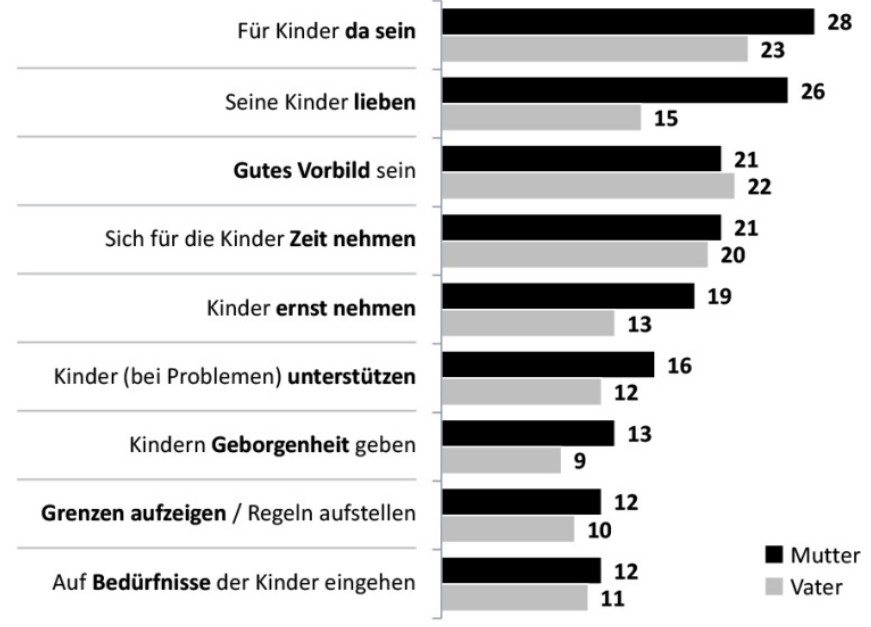

Abbildung 4: Beschreibung des Begriffs „gute Eltern“

Bei der Mutter ist der einzig relevante Unterschied zwischen Tochter und Sohn bereits erläutert worden. Beim Vater spielt es bei zwei Aspekten eine Rolle, ob parallel die Tochter oder der Sohn befragt wurde. „Kinder ernst nehmen“ wird vom Vater leicht häufiger genannt bei paralleler Befragung des Sohnes, während „Grenzen aufzeigen/Regeln aufstellen“ eher bei Töchtern häufiger genannt wird. Väter haben zu ihren Töchtern häufig eine etwas speziellere Beziehung, da vermutlich verstärkt das Bedürfnis auftritt, die Tochter beschützen zu müssen. Dies kann gegebenenfalls das etwas häufigere Nennen von „Grenzen aufzeigen/Regeln aufstellen" erklären.

Es lässt sich konstatieren, dass „gute Eltern“ vor allem eins für ihre Kinder machen müssen: Da sein. Dies klingt einfach, ist aber wahrscheinlich im Alltag nicht immer leicht umsetzbar. Eltern haben in diesem Zusammenhang die höchsten Ansprüche an sich selbst, denn eines wollen sie mit Sicherheit sein: gute Eltern. Inwieweit dies von den Kindern wahrgenommen wird, wird im folgenden Abschnitt in Ansätzen aufgezeigt. 


\section{Zufriedenheit mit gemeinsamer Zeit}

Der Begriff der Zufriedenheit kann viele Bestandteile umfassen. Im Zusammenhang mit dem Thema „Gemeinsame Zeit von Kindern und ihren Eltern“ zählen aus Sicht der Eltern hierzu die bisher genannten Aspekte: Wie viel Zeit wird gemeinsam verbracht? Wie wird diese gestaltet? Wie wird sie wahrgenommen und bewertet? Es stellt damit eine übergeordnete Einstellung dar. Besonders bei jüngeren Kindern ist gegebenenfalls nicht das übergeordnete Konstrukt der Zufriedenheit im Antwortverhalten berücksichtigt worden. Aber durch die Fragestellung „Wie zufrieden bist du damit, wie deine Eltern für dich da sind?“ wurde genau auf den Hauptaspekt zurückgegriffen, der bei den Eltern im Zusammenhang mit der Beschreibung von „guten Eltern“ widergegeben wurde - nämlich, dass Eltern für ihre Kinder da sei müssen.

Werden die Antworten der Kinder mit den Antworten der Eltern gegenübergestellt, zeigt sich im Gegensatz zu vielen der bisherigen Darstellungen eine recht unterschiedliche Wahrnehmung der einzelnen Gruppen. Allerdings passen auch diese übergeordneten Empfindungen ins bisherige Bild, das von den einzelnen Familienmitgliedern gezeichnet wurde. Mehr als jedes zweite Kind (53\%) gibt an, sehr zufrieden damit zu sein, wie seine Eltern für es da sind. Weitere $43 \%$ beschreiben die Situation mit „meistens zufrieden“. Lediglich $4 \%$ der Kinder geben an, oft nicht zufrieden zu sein. Dieser Wert erscheint zwar sehr niedrig, sollte aber doch zu denken geben, denn somit ist jedes 20. Kind zwischen sechs und 14 Jahren damit unzufrieden, wie seine Eltern für es da sind. Es zeigen sich keine relevanten Unterschiede in der Bewertung, je nachdem ob das Kind eine Ganztagsschule besucht, ob es Geschwister hat oder Einzelkind ist. Interessanterweise liegen aber relevante Unterschiede zwischen Jungen und Mädchen vor: Während bei den Mädchen $50 \%$ angeben, sehr zufrieden zu sein, sind es unter den Jungen sogar 57\%. Da die Angaben der Mütter und Väter nicht darauf hindeuten, dass tatsächlich in irgendeiner Art und Weise mehr Zeit mit Jungen als mit Mädchen verbracht wird, könnte vermutet werden, dass Jungen nicht ganz so viel Aufmerksamkeit benötigen wie Mädchen. Dies kann auf Grundlage der vorliegenden Datenbasis jedoch nicht zweifelsfrei belegt werden. In jedem Fall sind Mädchen nicht direkt unzufrieden mit der Zeit, die ihre Eltern mit ihnen verbringen, denn ihr Anteil steigt entsprechend bei der Antwort „meistens zufrieden“. Somit sind Mädchen vielleicht nur etwas kritischer als Jungen. Genauso zeigen sich leichte Unterschiede im Alter der Kinder. Bei den 6-10-Jährigen sind 56\% sehr zufrieden, bei den 11-14-Jährigen sind es 50\%. Aber auch dies gleicht sich nahezu wieder aus mit der folgenden Antwortoption ,meistens zufrieden“. Hier sind es $40 \%$ der 6-9-Jährigen und $44 \%$ der 11-14-Jährigen. Ähnlich wie die Mädchen scheinen auch die älteren Kinder etwas kritischer zu sein. Eine andere Überlegung könnte sein, dass die 11-14-Jährigen gar nicht mehr so viel Zeit mit ihren Eltern verbringen möchten, sondern lieber mit Freunden unterwegs wären o.ä. Dann rührt die Unzufriedenheit eher daher, dass sie aus ihrer Sicht zu viel Zeit mit ihren Eltern verbringen sollen. Inwieweit dies mit in die Bewertung hineinspielt, kann nicht final unterschieden werden.

Bei einer etwaigen Berufstätigkeit der Mutter zeigt sich ein interessantes Bild: Wenn die Mutter als Hausfrau zu Hause ist oder in Teilzeit arbeitet, liegt der Anteil der Kinder, die sehr zufrieden mit der gemeinsamen Zeit sind, bei $58 \%$. Erst wenn die Mutter in Vollzeit berufstätig ist, sinkt der Anteil auf $44 \%$. Erneut zeigt sich, dass erst bei einer Berufstätigkeit in Vollzeit Unterschiede in der Bewertung auftauchen. Aber es muss ergänzt werden, dass auch die Kinder mit einer in Vollzeit berufstätigen Mutter nicht direkt unzufrieden sind, denn sie geben entsprechend häufiger an, zumindest „meistens“ zufrieden zu sein. Kinder, die bei alleinerziehenden Müttern aufwachsen, zeigen eine ähnliche Verteilung. 
Auch hier sinkt der Anteil derer, die „sehr zufrieden“ sind zugunsten von ,meistens zufrieden" leicht. Dies wird aber natürlich auch stark davon getrieben, dass der Anteil der in Vollzeit berufstätigen Mütter bei Alleinerziehenden höher ausfällt als bei Paarhaushalten.

\section{Kinder / Mütter / Väter Gesamt - Zufriedenheit mit Menge an gemeinsamer Zeit Angaben in \%}

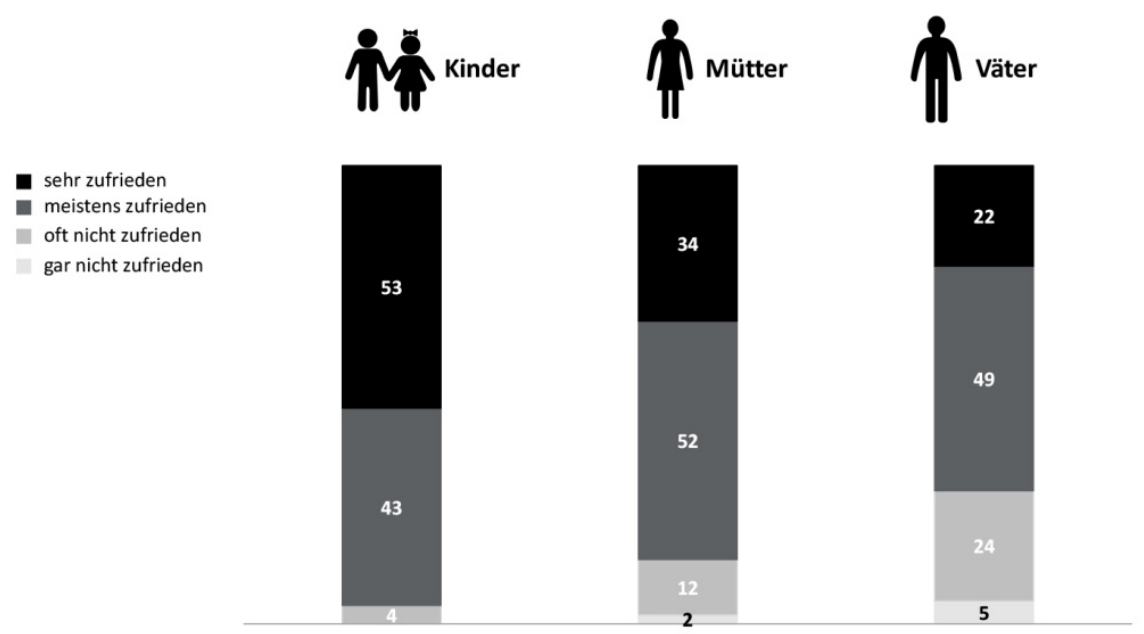

Basis: alle Kinder $n=1.012$; alle Mütter $n=1.002$; alle Väter $n=846$; geschlossene Frage

Abbildung 5: Zufriedenheit mit gemeinsamer Zeit

Wird der Fokus der Betrachtung auf die Mutter und den Vater gelegt, zeigt sich in der Summe eine sehr viel kritischere Wahrnehmung: Ein Drittel der Mütter (34\%) sind mit der Menge an gemeinsamer Zeit mit den Kindern sehr zufrieden. Weitere 52\% sind zumindest meistens zufrieden, während $12 \%$ oft nicht zufrieden und $2 \%$ gar nicht zufrieden sind. Damit liegt der Unterschied bei den Kindern und den Müttern innerhalb der Bewertung „sehr zufrieden“ bei 19 Prozentpunkten. Wird die Antwortoption ,meistens zufrieden“ mit in die Betrachtung einbezogen, liegt der Unterschied immer noch bei zehn Prozentpunkten. An einigen Stellen ist bereits darauf hingewiesen worden, dass Erwachsene in der Beurteilung von Dingen häufig sehr viel kritischer als Kinder sind. Daher ist eine Differenz in der Bewertung an dieser Stelle nicht verwunderlich. Nichtsdestotrotz können diese Unterschiede jedoch als Indiz dafür dienen, dass die Mutter an vielen Stellen mit einem schlechten Gewissen kämpft, weil sie das Gefühl hat, nicht genügend Zeit mit ihrem Kind zu verbringen. Diese Wahrnehmung kann aus Sicht des Kindes nicht bestätigt werden, da die Kinder zum großen Teil sehr zufrieden mit der Zeit sind, die ihre Eltern für sie haben.

Erwartungsgemäß sehen die Angaben der Mütter, wenn sie alleinerziehend oder berufstätig sind, erneut anders aus. Gerade der Anteil derer, die mit der gemeinsamen Zeit sehr zufrieden sind, ist bei alleinerziehenden Müttern weitaus niedrigerer. Hier sind $25 \%$ sehr zufrieden, während $58 \%$ meist zufrieden sind. Wenn die Mutter mit dem Vater oder einem Partner zusammenlebt, sind 36\% sehr zufrieden und 51\% meistens zufrieden. Weitaus größer fällt der Unterschied aus, wenn die Mutter berufstätig ist: Hier sind $26 \%$ sehr zufrieden, und $57 \%$ meistens zufrieden. Wenn die Mutter Hausfrau ist, sind hingegen 54\% 
sehr zufrieden und 39\% meist zufrieden. Somit gleicht sich hier das Verhältnis in der Top2-Box nicht komplett wieder aus. Immerhin $15 \%$ der berufstätigen Mütter sind oft nicht zufrieden, während es nur $7 \%$ der Hausfrauen sind. Und auch innerhalb der berufstätigen Mütter treten große Unterschiede auf: Bei den in Vollzeit berufstätigen Müttern sind $24 \%$ oft nicht zufrieden, während es bei den in Teilzeit berufstätigen Müttern $10 \%$ sind. Letztere weisen im Vergleich zu den in Vollzeit berufstätigen Müttern einen höheren Anteil mit der Antwort „meistens zufrieden“ auf. Es zeigt sich aber eindeutig, dass gerade berufstätige Mütter immer mit ihrem Zeitmanagement zu kämpfen haben. Aufgrund ihrer Berufstätigkeit haben sie regelmäßig das Gefühl, zu selten für ihre Kinder da zu sein. Genau das ist es jedoch, was für sie ,gute Eltern“ ausmacht, somit führt hier der empfundene Zeitmangel zu kontinuierlichen Gewissenskonflikten.

Im Vergleich zu den Müttern sind die Väter noch kritischer mit sich selbst, wobei dies stark damit zusammenhängt, dass der Großteil der Väter eben auch in Vollzeit berufstätig ist. Nur ein Fünftel (22\%) geben an, sehr zufrieden mit der Menge an gemeinsamer Zeit mit ihren Kindern zu sein. Weitere $49 \%$ sind zumindest meistens zufrieden, aber immerhin ein Viertel (24\%) sind damit oft nicht zufrieden. Und 5\% sind sogar gar nicht zufrieden. Somit schließt sich auch hier der Kreis: Die Kinder haben gerade bei der Beschreibung, warum die gemeinsame Zeit mit dem Vater vielleicht auch mal nicht so schön ist, darauf hingewiesen, dass der Vater oft zu wenig Zeit hat oder in dieser wenigen Zeit gestresst ist. Dies ist den Vätern auch sehr bewusst, denn sie selbst sind sehr kritisch in Bezug auf die Menge der gemeinsam verbrachten Zeit.

Somit ist der erste Schritt getan, da das Bewusstsein für die etwaigen Probleme auf beiden Seiten vorhanden ist. Inwieweit die Situation verbessert werden kann, ist aber fraglich, da im Berufsleben natürlich auch immer größere Ansprüche an Arbeitnehmer - egal ob mit Kindern oder ohne - gestellt werden. Somit werden Berufstätige immer den Spagat schaffen müssen, ihrer Arbeit nachzugehen und gleichzeitig in ausreichendem Maße für ihre Kinder da zu sein. Es kann nur erneut darauf hingewiesen werden, dass die Antworten der Kinder zwar ab und an darauf hinweisen, dass sie das Gefühl haben, ihre Eltern hätten durch die Arbeit weniger Zeit für sie. Aber sie sind insgesamt doch sehr zufrieden mit der Zeit, die ihre Eltern für sie da sind. Denn natürlich haben auch die Kinder nicht nur im Blick, dass ihre Eltern Zeit mit ihnen verbringen, sondern dass sie eben auch arbeiten gehen müssen bzw. wollen.

Dieses Verständnis zeigt sich, wenn man Kinder nach der Berufstätigkeit ihrer Eltern fragt. Knapp über die Hälfte der Kinder (56\%) stimmen der Aussage total zu, dass sie froh sind, dass ihre Mutter eine Arbeit hat. Beim Vater sind es $63 \%$. Werden Antworten mit „stimme eher zu“ hinzugenommen, sind es bei der Mutter $92 \%$ und beim Vater $91 \%$. Dieser Anteil fällt zudem höher aus, wenn die Kinder älter werden. Nahezu allen Kindern ist bewusst, dass Eltern einer Arbeit nachgehen müssen und sie sind froh, dass ihre Eltern das können. Dies wird ihnen von den Eltern bewusst gemacht: $90 \%$ bzw. $91 \%$ der Mütter und Väter stimmen der Aussage „Ich finde es wichtig, meinem Kind/meinen Kindern vorzuleben, dass es für eine/n Frau/Mann und Mutter/Vater normal ist, zu arbeiten“ total bzw. eher zu. Und natürlich sind die Eltern auch dankbar, dass sie Arbeit haben und nehmen daher Einschränkungen dem Kind gegenüber in Kauf. Dies bestätigen $70 \%$ der Väter und $57 \%$ der Mütter. Hier zeigen sich Unterschiede zwischen den Geschlechtern, da sich Frauen offenbar eher an ihre Mutterrolle gebunden fühlen und ihr Kind durch die eigene Berufstätigkeit in keinerlei Hinsicht benachteiligen möchten. Der Anteil der zustimmenden Mütter steigt auf $66 \%$ an, wenn die Mutter in Vollzeit berufstätig ist.

Ein ähnliches Bild zeigt sich, wenn man danach fragt, ob die Elternteile es schade finden, dass man wegen der Arbeit nicht so häufig für das Kind da sein kann, wie man möchte. Hier stimmen $64 \%$ der in Vollzeit berufstätigen Mütter zu, während es bei den in 
Teilzeit berufstätigen Müttern 36\% sind. Die Unterschiede zwischen Vollzeit- und Teilzeitstelle werden erneut offensichtlich: Eine Arbeitsstelle in Teilzeit scheint sich noch sehr gut mit der Mutterrolle vereinbaren zu lassen. Sobald die Mutter voll berufstätig ist, wird es sehr eng mit der Zeit, die es zu verteilen gilt, und es treten verstärkt Gewissenskonflikte auf. Bei den Vätern finden es vergleichbare $69 \%$ schade, dass sie wegen der Arbeit nicht so viel Zeit mit ihrem Kind verbringen können. Analog zur in Vollzeit berufstätigen Mutter offenbart sich auch hier der innere Konflikt, in dem sich berufstätige Eltern regelmäßig befinden. Aber auch hier zeigt sich, dass das schlechte Gewissen bei den Eltern oft größer ist als es sein müsste. Der Aussage, ob sie es schade finden, dass ihre Mutter bzw. ihr Vater wegen der Arbeit oft nicht für sie da ist, stimmen lediglich $28 \%$ der Kinder im Zusammenhang mit ihrer berufstätigen Mutter - unabhängig davon ob die Mutter in Vollzeit oder in Teilzeit arbeitet - zu. Beim Vater ist es schon jedes zweite Kind, aber dies schien bereits an anderen Stellen durch. Da der Vater insgesamt sehr häufig hinter der Mutter zurücktritt, was gemeinsame Zeit mit dem Kind angeht, ist es nicht verwunderlich, dass Kinder sich mehr Zeit mit ihrem Vater wünschen.

Immerhin zwei von drei berufstätigen Müttern bestätigen, dass es schwierig ist, gleichzeitig Beruf und Haushalt gut zu bewerkstelligen. Unter den in Vollzeit Beschäftigten ist es sogar jede dritte von vier Müttern. Gleichzeitig sagen 70\% der Mütter, dass das Kind/die Kinder schneller selbstständig werden muss/müssen, da sie selbst arbeitet und sich nicht um alles kümmern kann. Es entsteht eine andere Orientierungsphase. Es wurde viel über das schlechte Gewissen der berufstätigen Mütter gesprochen. Aber der Aussage, dass man trotz Berufstätigkeit und auch wenn mal nicht alles hundertprozentig perfekt läuft, trotzdem das Gefühl hat, eine gute Mutter zu sein, stimmen $93 \%$ der Mütter zu. Sie wissen, dass mit Sicherheit einiges besser und anders laufen sollte/könnte, aber das gibt ihnen nicht das grundsätzliche Gefühl, eine schlechtere Mutter zu sein. Sie wissen, welche wichtige Rolle sie für ihre Kinder einnehmen und fühlen sich in diesem Bereich auch selbst bestätigt.

Die Frage, ob berufstätige Mütter in Deutschland immer noch ein schlechtes Ansehen haben, bejahen lediglich $36 \%$ der berufstätigen Mütter. Der Anteil steigt leicht auf $43 \%$, wenn die Mutter in Vollzeit berufstätig ist. Ebenso geben lediglich $16 \%$ der berufstätigen Mütter an, dass sie sich für ihre Wahl oft vor Hausfrauen rechtfertigen müssen. Auch aus der Vatersicht wird Unterstützung widergespiegelt: $72 \%$ der Väter geben an, dass ihnen die Berufstätigkeit ihrer Frau am Herzen liegt und sie versuchen, sie darin zu unterstützen. Es scheint, dass die Berufstätigkeit von Müttern immer mehr „normal“ wird. Als etwas schwieriger wird da die Rolle des Vaters gesehen: Der Aussage, dass in Deutschland Väter, die für ihre Kinder beruflich kürzertreten, immer noch ein schlechtes Ansehen haben, stimmen immerhin mehr als die Hälfte der berufstätigen Väter zu (58\%). Inwieweit es Vätern heutzutage ermöglicht wird, sich neben ihrer Berufstätigkeit in Vollzeit Zeit für die Familie und besonders ihre Kinder zu nehmen, um eben auch den Erwartungen der Kinder an sie gerecht zu werden, stellt ein neues, aktuelles Thema dar, dass es an anderer Stelle aus zusätzlichen Blickwinkeln zu beleuchten gilt.

Zum Abschluss soll der Fokus noch einmal im Detail auf das Gesamtkonstrukt der Familie gelegt werden, welches bisher von den relevanten Personengruppen der Mütter, Väter und Kinder in vielen Einzelbestandteilen beleuchtet wurde. Wenn alle drei Gruppen danach befragt werden, wie zufrieden sie mit dem gemeinsamen Familienleben sind, zeigt sich erneut ein sehr positives und auch übereinstimmendes Bild. Mehr als die Hälfte der Kinder (51\%) ist „sehr zufrieden“ damit. Wird die Einschätzung „meistens zufrieden“ hinzugenommen, werden bereits $94 \%$ der Kinder berücksichtigt. Während es zwischen Jungen und Mädchen keine Unterschiede im Antwortverhalten gibt, sind die jüngeren Kinder zwischen 6-10 Jahren erneut noch positiver: 55\% sind „sehr zufrieden“, 39\% „meistens zufrieden“. Bei den älteren Kindern zwischen 11-14 Jahren sind $45 \%$ „sehr zufrieden“ und $48 \%$ 
„meistens zufrieden“. Somit sind die älteren Kinder nicht besonders unzufrieden, sondern möglicherweise nur etwas kritischer. Noch kritischer sind aber die Eltern, die sich darin zudem sehr einig sind. 36\% der Mütter bzw. 37\% der Väter sind „sehr zufrieden“ mit dem Familienleben, weitere $55 \%$ bzw. 52\% „meistens zufrieden“. Die Eltern sind zudem nach ihren Beweggründen für diese Einschätzung befragt worden. Bei beiden Elternteilen, die „sehr zufrieden“ sind, zeichnen sich ähnliche Gründe ab. Am häufigsten wird auf das ,harmonische Miteinander“ verwiesen. Mit Aspekten wie „wir sind ein Team“, „wir vertrauen uns" oder „wir lieben uns" spielen weitere gefühlsbetonte, zwischenmenschliche Gründe eine große Rolle. Aber auch die konkrete gemeinsame Zeit, verbunden mit Erlebnissen, macht ein zufriedenstellendes Familienleben aus, wie Aspekte wie z.B. „etwas gemeinsam unternehmen/viele gemeinsame Erlebnisse haben“ oder „wir genießen die gemeinsame Zeit" zeigen.

Bei den wenigen Müttern, die mit dem Familienleben „oft nicht zufrieden“ sind, spielt die wenige gemeinsame Zeit eine große Rolle. $15 \%$ geben an, dass ihr Partner oft zu wenig Zeit für die Familie aufbringt. Weitere $10 \%$ hätten gerne selbst mehr Zeit für das Familienleben. Bei den Vätern nennen sogar 19\% diesen Grund. Bei beiden Elternteilen bemängeln $22 \%$ ganz grundsätzlich, dass es viel zu wenig Zeit gibt, die gemeinsam als komplette Familie miteinander verbracht wird. Während $12 \%$ der Mütter als Grund angeben, dass sie alleine für die Erziehung und den Haushalt zuständig sind, zeigt sich bei den Vätern wieder der Druck durch die Berufstätigkeit in Vollzeit. 17\% sagen, dass aufgrund von anderen Verpflichtungen zu wenig Zeit da ist. Weitere $12 \%$ beziehen dies speziell auf den Zeitraum unter der Woche, in dem sie durch ihre Berufstätigkeit stark eingebunden sind.

\section{Kinder / Mütter / Väter Gesamt - Zufriedenheit mit dem Familienleben \\ Angaben in \%}
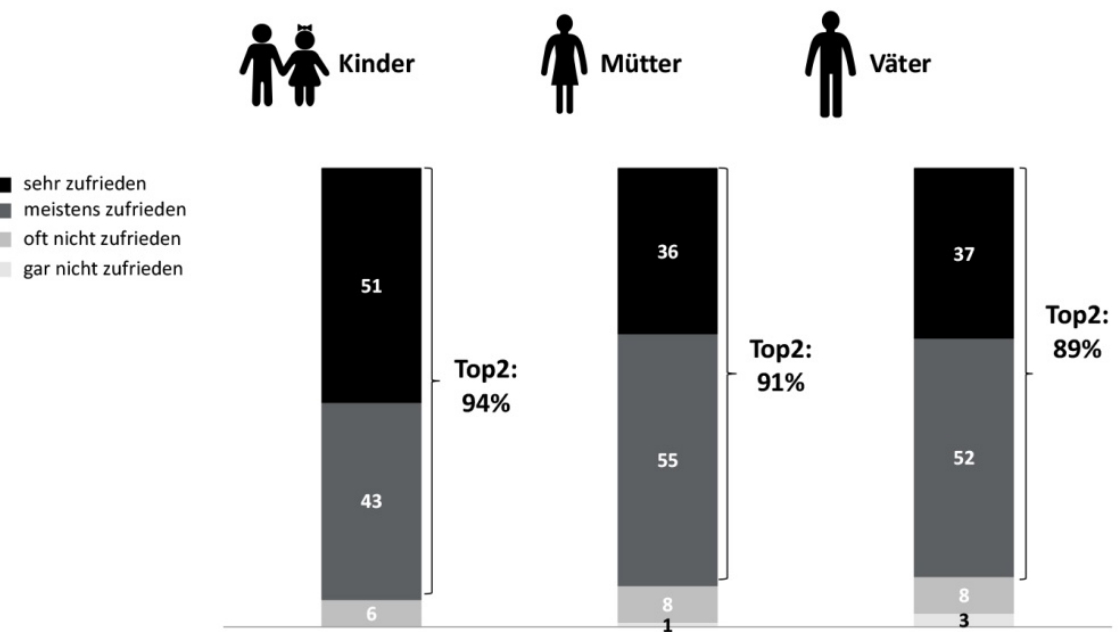

Basis: alle Kinder $n=1.012$ Kinder; alle Mütter $n=1.002$; alle Väter $n=846$; geschlossene Frage

Abbildung 6: Zufriedenheit mit dem Familienleben 


\section{Fazit}

Die gemeinsame Zeit von Kindern und ihren Eltern ist aus vielen verschiedenen Blickwinkeln betrachtet worden. Es konnte gezeigt werden, dass für die meisten Kinder die Mutter immer noch die Bezugsperson ist, mit der am meisten Zeit verbracht wird. An einzelnen Stellen zeigt sich, dass die Kinder auch mit ihren Vätern sehr gerne viel mehr Zeit verbringen würden. Gleichzeitig haben die Kinder aber auch ein großes Verständnis dafür, dass ihre Väter bzw. ihre Eltern generell arbeiten gehen müssen und dadurch weniger Zeit zur Verfügung steht. Umso schöner ist es, dass eindeutig eine positive Wahrnehmung der Menge an gemeinsamer Zeit von allen Gruppen festzuhalten ist. Unter der Woche wird dies zwar etwas kritischer beschrieben als am Wochenende, aber trotzdem zeigt sich ein absolut positives Bild, sodass die gemeinsame Zeit zwischen Kindern und ihren Eltern durchweg als viel bzw. ausreichend beschreiben werden kann. Ebenso ist der Großteil der gemeinsamen Zeit sowohl für die Kinder als auch für die Eltern meistens schön. Dies wird bei den Kindern vor allem an gemeinsamen Aktivitäten/Erlebnissen festgemacht. Wenn die Kinder etwas älter werden, spielen auch zwischenmenschliche Gründe, wie das Gefühl, dass die Eltern dem Kind den Rücken stärken, eine größere Rolle. Erst wenn der Fokus auf die übergeordnete Zufriedenheit mit der gemeinsamen Zeit gelegt wird, zeigt sich bei den Eltern ein schlechtes Gewissen. Während Kinder auf einem hohen Niveau zufrieden sind, sind die Eltern durchaus kritischer mit sich - gegebenenfalls sogar zu kritisch. Gerade bei berufstätigen Eltern schwingt immer das Gefühl mit, aufgrund des Berufs zu wenig Zeit mit dem Kind zu verbringen, was bei berufstätigen Eltern zu einem inneren Konflikt führt. Es sollte aber in den Vordergrund gestellt werden, dass die Kinder ein insgesamt sehr positives Bild von der gemeinsamen Zeit mit ihren Eltern zeichnen.

\section{Literatur}

UNICEF (2006, 2008, 2010): Kinderwertemonitor 2006/2008/2010. Forschungsbericht (unveröff.).

UNICEF (2010): Geolino-Kinderwertemonitor 2010: „Vertrauen“ und „Respekt“ gewinnen für Kinder an Bedeutung. https://www.unicef.de/presse/2010/kinderwerte-monitor/29156 [Zugriff: 24.06.2016] 